\title{
Self-Organizing Map Classification of the Extremely Low-Frequency Magnetic Field Produced by Typical Tablet Computers
}

\author{
Darko Brodić $^{1}$, Alessia Amelio², Ivo R. Draganov ${ }^{3}$ \\ ${ }^{1}$ University of Belgrade, Technical Faculty in Bor, 19210 Bor, Serbia, dbrodic@tfbor.bg.ac.rs \\ ${ }^{2}$ DIMES, University of Calabria, 87036 Rende (CS), Italy, aamelio@dimes.unical.it \\ ${ }^{3}$ Faculty of Telecommunications at Technical University of Sofia, Sofia 1000,Bulgaria, idraganov@tu-sofia.bg
}

\begin{abstract}
In this paper, the extremely low frequency magnetic field produced by the tablet computers is explored. The measurement of the tablet computers' magnetic field is performed by using a measuring geometry previously proposed for the laptop computers. The experiment is conducted on five Android tablet computers. The measured values of the magnetic field are compared to the widely accepted TCO safety standard. Then, the results are classified by the Self-Organizing Map method in order to create different levels of safety or danger concerning the magnetic field to which tablet computer users are exposed. Furthermore, a brief comparison of the obtained magnetic field levels with the ones from typical laptops is performed. At the end, a practical suggestion on how to avoid the high exposure to the low frequency magnetic field emitted by the tablet computers is given.
\end{abstract}

Keywords: Classification, magnetic field, measurement, Self-organizing map, tablet computers.

\section{INTRODUCTION}

The artificial magnetic field is a byproduct of the electric current, which flows through electrical or electronic devices. Accordingly, the magnetic field is present everywhere in our environment. It is characterized by its frequency. The time varying magnetic field produced by electrical gadgets contains an Extremely Low Frequency (ELF) component. The ELF magnetic field typically includes frequencies from $30 \mathrm{~Hz}$ to 300 $\mathrm{Hz}$. The magnetic field is strong if it is close to the emitter. Also, it rapidly decreases in the case of extremely low frequencies if we are getting away from the source. It is worth noting that some materials can block or rapidly decrease the level of magnetic field spreading.

A magnetic field with different frequencies can interact with the human body creating internal currents [1]. Although it is not destructive as the ionizing radiation, such as gamma or x-rays, it has some potential negative effects on humans. At extremely low frequencies, the levels of induced currents inside the body are too small to produce meaningful effects. However, such magnetic field induces currents within the human body, which can be sufficient to produce a variable effect on humans during long work. Also, there is no doubt that a short-term exposure to very high levels of magnetic field can be harmful to the human health. The current public concern focuses on possible long-term health effects caused by an exposure to magnetic fields at levels below those required to trigger acute biological responses. Up until now, the exten- sive research in many studies, which have been conducted in the area of the ELF magnetic field, has not given a clear answer on how it is harmful to human health. With more and more available research expertise, it has become increasingly likely that an exposure to magnetic fields determines serious health hazards. Nevertheless, some uncertainties remain. The original scientific discussion about the interpretation of controversial results has shifted to become a societal as well as an economic issue.

International safety standards concerning the magnetic fields have been developed to establish the level of exposure to the magnetic field which is not harmful to the human health. Accordingly, the standards for low frequency magnetic fields ensure that induced electric currents are below the normal level of background currents within the body. During the measurement of low frequency magnetic fields, the magnetic flux density has been characterized in terms of field amplitude and WP index [2], [3], that implement the weighted peak approach, well-suited in the case of complex waveforms. The security limits for exposure to a magnetic field are different according to different authors: $0.2 \mu \mathrm{T}$ [4], $0.4 \mu \mathrm{T}$ [5], and $1 \mu \mathrm{T}$ [6]. Also, an exposure to $1 \mu \mathrm{T}$ of magnetic field reduces the total sleep time as well as sleep efficiency [6]. The widely accepted TCO standard proposes the following: (i) a prescribed geometry for the measurement of the magnetic field (measured at $0.30 \mathrm{~m}$ in front of and around the emitter) and (ii) a test procedure with reference values of the magnetic 
flux density in the frequency region between $5 \mathrm{~Hz}$ and $2 \mathrm{kHz}$ [7].

A tablet computer, tablet for short, represents a mobile computer, which includes a touchscreen display, circuitry, and battery. Also, it is equipped with sensors, cameras, a microphone, and an accelerometer. The touchscreen display typically uses finger recognition to replace the mouse and keyboard. The keyboard is given as a pop-up virtual keyboard which is used for typing. The tablet can also include some physical buttons. They are used for basic features such as speaker volume and power, and ports for network communications and battery charging. The tablet is usually characterized by screen size. It is supposed that the tablets incorporate a screen from 7" to 10 " wide.

In this paper, we pay special attention to the ELF magnetic field produced by the tablets. Hence, an experiment is conducted in order to measure, evaluate, and classify the ELF magnetic field to which the users are exposed. Accordingly, the high frequency magnetic field from the tablets' components like WIFI, $3 \mathrm{G} / 4 \mathrm{G}$ or similar is out of the scope of this paper. To the best of our knowledge, this is one of the first studies that analyze this problem. It has great importance because of the wide-spreading of the tablets among the younger population. It is worth noting that the experiment is conducted on typical tablets. It means that we tested 5 tablets with screens from 7" to 10" wide, working in the "normal" operating condition. This condition means that the tablets are only used for Internet browsing. The tested tablets operate under the most widely spread tablet operating system, i.e. Android. In this way, we test the tablets in similar or the same conditions. The tablets are usually made of gorilla glass material at their top, and of plastic material at their bottom. At the end, we make the assumption that the users' hands are in close contact with the touch area as well as its back. Hence, we accustom the measuring geometry to this way of using the tablets.

The rest of the paper is organized as follows. Section 2 describes the elements of the measuring method, the measuring equipment, the proposed measuring geometry, and the experiment. Section 3 gives the results of the measured ELF magnetic field. Section 4 explains the classification technique, which will be used for the classification of the obtained measurement results. Section 5 presents the classification of the measurement results in order to establish the dangerous and safe levels of the magnetic field. At the end, Section 6 draws the conclusions.

\section{Methods And Materials}

\subsection{Magnetic fields}

In the normal operating condition, the inner components of the tablet are supplied by a current $I$. In this way, the current $I$ flows through these electronic or electrical components inducing a magnetic field. This magnetic field is registered and measured by measuring devices. Usually, these devices register the scalar components of the magnetic induction: $B_{x}$, $B_{y}$, and $B_{z}$ as well as the Root Mean Square (RMS) of the magnetic induction $B$, which is calculated as:

$$
B=\overline{B_{x}+B_{y}+B_{z}} .
$$

\subsection{Measuring devices}

The magnetic field measurement is performed by the measuring device Lutron EMF-828 with separate probe, which includes the sensing head as well as the spectrum analyzer AARONIA NF-5030.

Lutron EMF-828 measures the scalar components of the magnetic induction $B_{x}, B_{y}$, and $B_{z}$ in the range between 0.01 $\mu \mathrm{T}$ and $2 \mathrm{mT}$. It is measured in the ELF range, i.e. between 30 and $300 \mathrm{~Hz}$. The device has three measurement extents: $20 \mu \mathrm{T}, 200 \mu \mathrm{T}$, and $2 \mathrm{mT}$. The precision of the measurement is of the order of $0.01 \mu \mathrm{T}$ for the measurement extent of 20 $\mu \mathrm{T}, 0.1 \mu \mathrm{T}$ for the measurement extent of $200 \mu \mathrm{T}$, and $1 \mu \mathrm{T}$ for the measurement extent of $2 \mathrm{mT}$. Also, the measurement is confirmed by the measuring device AARONIA NF-5030. This device registers the minimum, maximum, average, and RMS magnetic induction in the range between $1 \mathrm{pT}$ and $2 \mathrm{mT}$. It covers the full ELF range between $1 \mathrm{~Hz}$ and $30 \mathrm{MHz}$. It has six predefined measurement extents in the given frequency range. However, each user can adjust the measuring device in order to register the magnetic field amplitude in a customdefined frequency measurement range.

\subsection{The measuring geometry}

The method consists of measuring the ELF magnetic field produced by the tablet computers. The measuring geometry consists of 22 measurement points on the surface of and around the tablet. It is an integration of the previously proposed measuring geometry for portable computers [8], [9] and an improvement of the measuring geometry proposed in the TCO standard [7]. The magnetic field is measured: (i) at nine different positions at the tablet touchscreen at the top, (ii) at nine different positions at the bottom of the tablet, and (iii) at four positions around the tablet $(30 \mathrm{~cm}$ away from the edge of the tablet). This measuring geometry is chosen as a considerable extension to the TCO proposed measuring geometry [7]. We have strong reasons to accept the new proposed measuring geometry because of the typical way of using the tablet. Usually, the tablet users are in direct and constant contact with the top and the bottom part of the tablet with their fingers. Hence, the geometry proposed by TCO does not give a real condition of the exposure of the tablet users to the magnetic field. Fig. 1 shows the measuring positions at the top and bottom of the tablet computer.

\subsection{Experiment}

The experiment was conducted on 5 different tablet computers. All tablets use the Android operating system. The tablets are tested in their typical working condition, i.e. during Internet browsing. The magnetic field is measured ten times at the measuring positions: (i) at the top: tbmp1 ... tbmp9 (top body measurement point - tbmp), (ii) at the bottom: bbmp1 ... bbmp9 (bottom body measurement point - bbmp) [8], [9], 


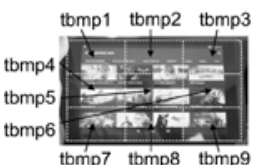

(a)

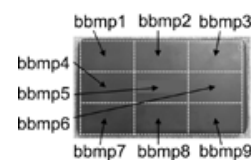

(b)

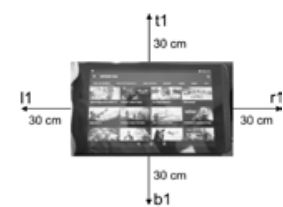

(c)
Fig. 1. Measuring positions of the tablet: (a) at the top (tbmp1,...,tbmp9), (b) at the bottom (bbmp1,...,bbmp9), (c) $30 \mathrm{~cm}$ away from the edge of the tablet $(\mathrm{t} 1, \mathrm{~b} 1, \mathrm{l} 1, \mathrm{r} 1)$

and (iii) at four measuring positions away from the edge of the tablet (t1,b1,11,r1). The average values of the measurement are used as reference. Hence, we measure the magnetic field of the tablets at no distance from the touchscreen display or its bottom. We make this assumption taking into account the typical use of the tablet where the users' fingers are positioned on its top and/or bottom parts all the time. Fig. 2 shows a typical way of using a tablet.

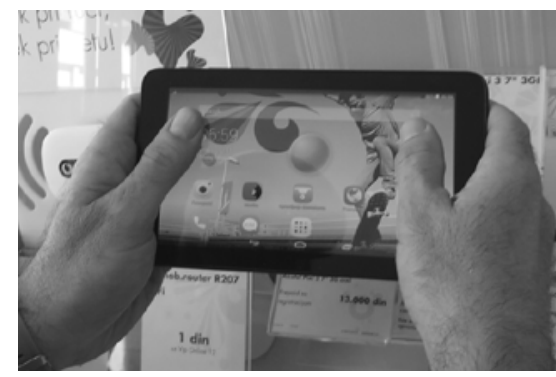

Fig.2. A typical way of using a tablet

\section{Measurement Results}

At the four measuring positions away from the edge of the tablet (t1,b1,l1,r1), the obtained magnetic field was below $0.02 \mu \mathrm{T}$. It can be considered as a background magnetic field. Hence, it is negligible.

The measurement results of the magnetic field for all tablets $\mathrm{Tj}$ where $\mathrm{j}=1, \ldots, 5$ are given in Fig. 3 .

Each area is characterised by a number representing the value of the emitted magnetic field in $\mu \mathrm{T}$. As a thresholding (dangerous) level, we used the proposed TCO reference level of $0.2 \mu \mathrm{T}$.

Table 1 shows the values of ELF magnetic field that are emitted by the top and bottom parts of the tablets in the minmax manner.

Table 1. Measured magnetic field at the top and bottom part of the tablet given in the min-max manner (in $\mu \mathrm{T}$ )

\begin{tabular}{|c|c|c|c|c|}
\hline Tablet & Top Min. & Top Max. & Bott. Min. & Bott. Max. \\
\hline T1 & 0.04 & 0.15 & 0.05 & 0.20 \\
T2 & 0.06 & 0.43 & 0.07 & 0.86 \\
T3 & 0.02 & 0.22 & 0.01 & 0.51 \\
T4 & 0.05 & 0.86 & 0.05 & 0.96 \\
T5 & 0.06 & 0.45 & 0.07 & 0.11 \\
\hline
\end{tabular}

From Table 1 (see max. values), we can see that all tablets have some dangerous areas, which emit a magnetic field value above the safety reference level. Also, 3 out of 5 tablets have dangerous values on both sides, i.e. at the top as well as bottom. Also, it is worth noting that the left and right side areas are the most exposed to the tablet users due to the typical way of using them.

\section{\begin{tabular}{|l|l|l|}
\hline 0.15 & 0.09 & 0.04 \\
\hline
\end{tabular} \begin{tabular}{|l|l|l|}
\hline 0.06 & 0.10 & 0.06 \\
\hline
\end{tabular} \begin{tabular}{l|l|l|}
\hline 0.05 & 0.08 & 0.04 \\
\hline
\end{tabular}}
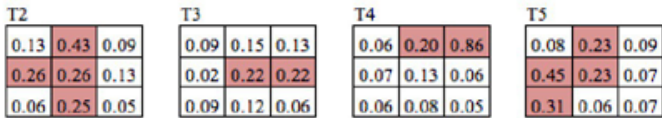

(a)
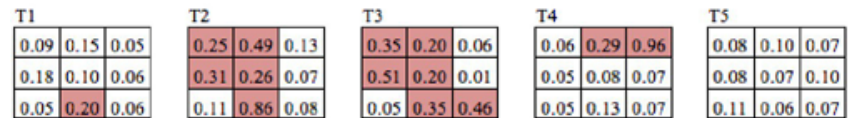

(b)

Fig. 3. Magnetic field measurement results given in $\mu \mathrm{T}$ : (a) measured values at the top areas of the tablets (touchscreen display area), (b) measured values at the bottom areas of the tablets. Colored fields represent the areas with higher magnetic field than it is allowed by the TCO standard $(\geq 0.2 \mu T)$ [7]

\section{Self-Organizing Map Classification}

In our analysis, we use a one-dimensional Self-Organizing Map (SOM) [10] to classify the measured values. It was selected because of its ability to adapt to the shape of the input data in order to manage a large variety of samples, and to cluster complex data sets in acceptable time. Furthermore, the SOM usually works fine in various applications, as it correctly finds the classification of the data points. Finally, the SOM revealed its potential in discretising numerical values in different contexts [11]. All these aspects make the SOM an invaluable model to be employed in multiple domains where other methods fail to accurately classify the input data. The adopted SOM is characterised by one input neuron and 5 output neurons. Fig. 4 illustrates the applied SOM.

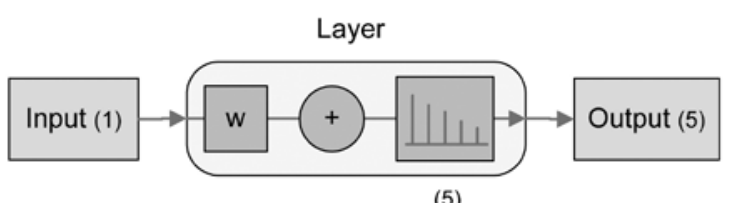

Fig.4. Illustration of the applied SOM structure

All neurons are initialized at the center of the feature space which corresponds to all the values from the minimum (typically 0 ) to a maximum of the magnetic induction $B$. For each experiment, the inputs are all 9 measured values for the given set of tablets at one of their sides, e.g., at the top and later at the bottom parts in their typical workload. The training of the network includes changes for the weights and bias by following the learning rules with incremental updates after each presentation of an input [12]:

$$
w_{i}(t+1)=w_{i}(t)+h_{c i}(t)\left[x(t)-w_{i}(t)\right] \text {, }
$$




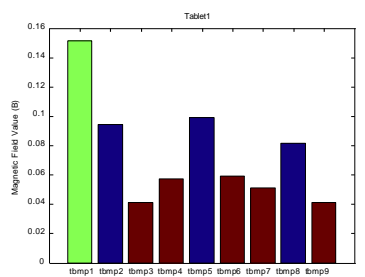

(a) $\mathrm{T} 1$

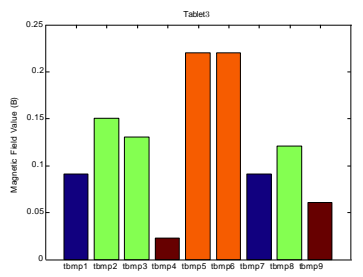

(c) $\mathrm{T} 3$

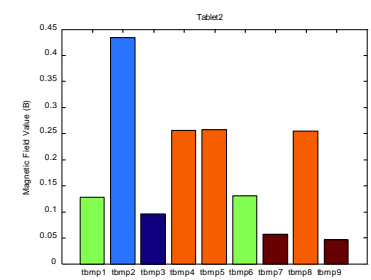

(b) $\mathrm{T} 2$

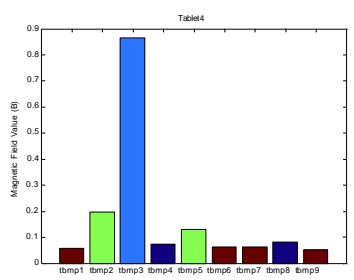

(d) T4

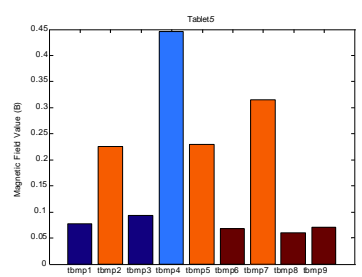

(e) T5

Fig. 5. Classification of the five tablets' magnetic field values at all nine measuring top positions (tbmp1...tbmp9)
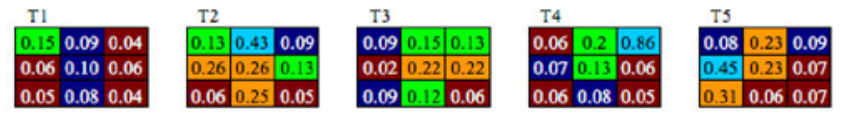

Fig.6. Dangerousness maps of the ELF magnetic field levels for the top parts of the measured tablets

where $w_{i}(t)$ is the $i$-th weight of the network at time $t ; h_{c i}$ the neighborhood function; $x$ - the current input vector, and $i=1 . . .5$.

The inputs are presented in random order for 1000 epochs in each simulation. Every training sample causes changes in the position of the nearest neuron, the most in accordance to the update value - an increment by which the neuron is moved towards the current position of the input. The Euclidean distance is used here as a measure [12]:

$c=\operatorname{argmin}_{i}\left\|x-w_{i}\right\|=\operatorname{argmin}_{i} \quad\left(\overline{x_{0}}-w_{i 0}\right)^{2}+\cdots+\left(x_{k}-w_{i k}\right)^{2}$

where $x$ and $w_{i}$ are one-dimensional for this particular realization. All other neurons change their position in a lighter degree. After reaching the end of the last epoch, the training is finished and the position of all neurons along the $B$ axis gives the cluster centroids. This is the final step of the clustering process.
In the next stage, which is the simulation of the network for every tablet at the given mode and side, all induction values are independently passed one by one through the input. Inside the SOM, the closest neuron is found for them by calculating the Euclidean distance to all 5 weights and selecting the minimal from them. A number from 1 to 5 is generated at the output denoting the associated class which the current test sample belongs to. Thus, the classification process takes place.

\section{Classification Results and Discussion}

Fig. 5 shows the classification results (5 classes) from all five tablets measured at the top positions. If we project the ELF magnetic field levels obtained by the classification into the measuring positions, then we can build a map of ELF magnetic field dangerousness levels. This map is shown in Fig. 6.

The ELF magnetic field levels are given in Table 2.

Table 2. The ELF magnetic field levels obtained by the SOM classification for the top parts of the tablets in the min-max manner (in $\mu \mathrm{T})$

\begin{tabular}{|l|c|c|c|}
\hline Magnetic field level & Dangerousness & Min. & Max. \\
\hline L1 (cyan) & Highly Dangerous & 0.43 & 0.86 \\
L2 (orange) & Dangerous & 0.22 & 0.31 \\
L3 (green) & Middle Safe & 0.12 & 0.20 \\
L4 (blue) & Safe & 0.07 & 0.10 \\
L5 (brown) & High Safe & 0.02 & 0.07 \\
\hline
\end{tabular}

From Table 2, we can see that the top part of the tablet emits two dangerous levels of ELF magnetic field, which are L1 and L2, and three safe levels of ELF magnetic field, which are L3, L4, and L5. The highest peak is $0.86 \mu \mathrm{T}$, which is much higher than the safety reference limit of $0.2 \mu \mathrm{T}$ [7].

From Fig. 5, we can observe that $40-60 \%$ of the top positions in most of the tablets emit Highly Dangerous and Dangerous levels or a Middle Safe level, which is a borderline level of values just below or equal to the safety reference limit of $0.2 \mu \mathrm{T}$.

From the top dangerousness maps of Fig. 6, we can notice that the Highly Dangerous, Dangerous, and Middle Safe levels mostly cover the areas where CPU and RAM are located. It can be observed in T4, where the Highly Dangerous and Middle Safe levels of ELF magnetic field are obtained in correspondence with the RAM, followed by the CPU, with values of $0.86 \mu \mathrm{T}, 0.2 \mu \mathrm{T}$ and $0.13 \mu \mathrm{T}$, respectively. Also, in T5 the RAM and CPU emit a Dangerous level, with values of $0.31 \mu \mathrm{T}$ and $0.23 \mu \mathrm{T}$, respectively. Also, in T1 the highest peak of ELF magnetic field is obtained in correspondence with the CPU, emitting a Middle Safe level with a value of $1.15 \mu \mathrm{T}$. Similar considerations can be performed for T2 and T3, where the areas emitting Highly Dangerous and Dangerous levels have a correspondence with the CPU and RAM, with the highest peaks of $0.43 \mu \mathrm{T}$ and $0.22 \mu \mathrm{T}$, respectively.

Fig. 7 shows the classification results ( 5 classes) for all five tablets measured at the bottom positions. The ELF magnetic field levels projected into the measuring positions provide the 


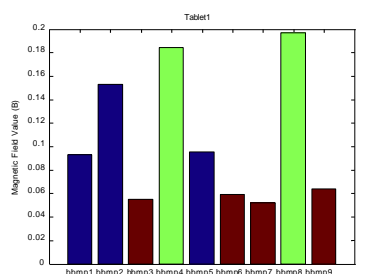

(a) T1

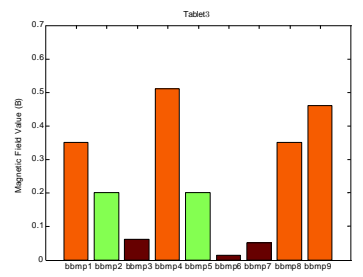

(c) T3

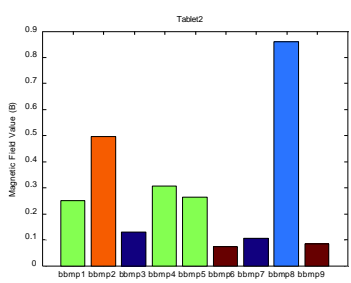

(b) $\mathrm{T} 2$

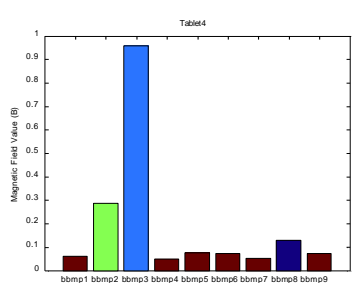

(d) $\mathrm{T} 4$

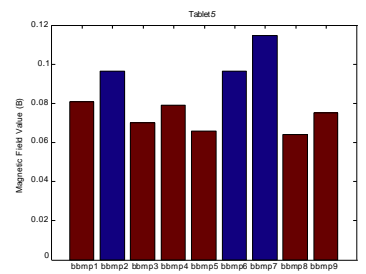

(e) $\mathrm{T} 5$

Fig. 7. Classification of the five tablets' magnetic field values at all nine measuring bottom positions (bbmp1...bbmp9)
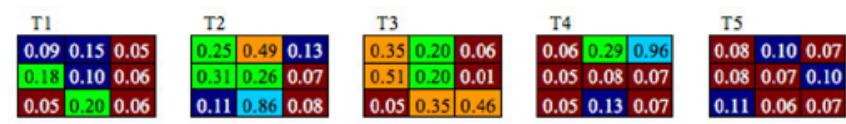

Fig.8. Dangerousness maps of the ELF magnetic field levels for the bottom parts of the measured tablets

dangerousness maps of the ELF magnetic field levels given in Fig. 8.

The ELF magnetic field levels are given in Table 3.

Table 3. The ELF magnetic field levels obtained by the SOM classification for the bottom parts of the tablets in the min-max manner (in $\mu \mathrm{T}$ )

\begin{tabular}{|l|c|c|c|}
\hline Magnetic field levels & Dangerousness & Min. & Max. \\
\hline L1 (cyan) & Highly Dangerous & 0.86 & 0.96 \\
L2 (orange) & Middle Dangerous & 0.35 & 0.51 \\
L3 (green) & Dangerous & 0.18 & 0.31 \\
L4 (blue) & Safe & 0.09 & 0.15 \\
L5 (brown) & High Safe & 0.01 & 0.08 \\
\hline
\end{tabular}

From Table 3, we can realize that the bottom part of the tablet emits three dangerous levels of ELF magnetic field, i.e. L1, L2, and L3, and only two safe levels of ELF magnetic field, i.e. L4 and L5. L3 is a borderline level, because it includes some values which are just below the safety reference limit of $0.2 \mu \mathrm{T}$ [7]. However, it is considered as a dangerous level, because most of the included values are above the reference limit of $0.2 \mu \mathrm{T}$. The highest peak of ELF magnetic field is $0.96 \mu \mathrm{T}$, which is noticeably higher than the safety reference limit of $0.2 \mu \mathrm{T}$.

Fig. 7 shows that two out of five tablets, i.e. T2 and T3, have $50-60 \%$ of positions emitting Highly Dangerous, Middle Dangerous or Dangerous levels. The other two tablets, T1 and T4 have 20\% of positions with Highly Dangerous or Dangerous levels. Finally, the last tablet, T5, safely emits at its bottom positions.

From the bottom maps in Fig. 8, it is confirmed that the positions emitting the highest dangerous levels are mostly associated with the RAM and CPU. It indicates that these components not only have an influence on the top part, but also on the bottom part of the tablet. On the contrary, it is interesting to observe that the battery has only an influence in ELF magnetic field emission on the bottom part of the tablet, because of its disposition in the tablet case. In particular, in T4 the position where the CPU is located emits a Highly Dangerous level, with a value of $0.96 \mu \mathrm{T}$, followed by the position where the RAM is located, emitting a Dangerous level, with a value of $0.29 \mu \mathrm{T}$. Also, in $\mathrm{T} 1$ a Dangerous level is obtained in the area where the battery is positioned, with the highest peak of $0.20 \mu \mathrm{T}$. Finally, T2 and T3 obtain Highly Dangerous, Middle Dangerous and Dangerous levels in the area of the CPU, RAM, and battery. In particular, in T2 the highest peak of ELF magnetic field is reached at the Highly Dangerous level, with a value of $0.86 \mu \mathrm{T}$. In T3, the highest peak is obtained at the Middle Dangerous level, with a value of $0.51 \mu \mathrm{T}$. In both cases, this peak is much higher than the safety reference limit of $0.2 \mu \mathrm{T}$.

A comparison between the top and the bottom ELF magnetic field levels shows that the bottom part emits higher dangerous levels than the top part. It is clearly visible from Tables 2 and 3. In fact, Highly Dangerous level is higher for the bottom part than for the top part of the tablet. Also, Middle Dangerous level is detected as a bottom range, but not detected as a top range. Dangerous level is very similar for the top and bottom parts. However, it is worth noting that the bottom part of the tablet adds a dangerous level (Middle Dangerous) which is not emitted at the top part of the tablet. On the contrary, Middle Safe level is emitted by the top part of the tablet (see Table 2), but it is not found at the bottom part of the tablet (see Table 3). Hence, a safe level is emitted at the top part, but not emitted at the bottom part. On the other hand, it is worth noting from Fig. 5 that most of the tablets have $40-60 \%$ of top positions with dangerous levels of ELF magnetic field. It is different from Fig. 7 where only 2 out of 5 tablets have $50-60 \%$ of bottom positions with dangerous levels. Nonetheless, in the last case, the ELF magnetic field value associated with these bottom positions is much higher than the value of the first case associated to the top positions.

From the aforementioned results, it is worth noting that the tablets do not emit the same level of measured magnetic field, which is emitted by the laptops [1], [8], [9], [13]. However, their intensity is still above the safety reference level proposed by TCO. To have a fair comparison between the tablets' and 
laptops' ELF magnetic field emission, we only have to compare the emitted magnetic field of the battery powered devices. In these conditions, the laptops emit an ELF magnetic field between $0.2 \mu \mathrm{T}$ and $4.5 \mu \mathrm{T}$ at the top body (case) parts and between $0.2 \mu \mathrm{T}$ and $3.5 \mu \mathrm{T}$ at the bottom body parts [8]. On the contrary, the tablets emit an ELF magnetic field at the top body parts between $0.02 \mu \mathrm{T}$ and $0.86 \mu \mathrm{T}$ and at the bottom body parts between $0.01 \mu \mathrm{T}$ and $0.96 \mu \mathrm{T}$.

Also, the comparison between tablets and laptops should take into account that the tablets have much less processing power than the laptops. Furthermore, they typically work as battery powered devices. Hence, an objective comparison can be only established with laptops working in battery mode. Also, it is worth noting that the tablets emit a higher level of magnetic field at their bottom parts compared to their top parts. This result is similar to the laptops. However, the reasons for such ELF magnetic field emission are different in laptops and tablets. The primary reason for a higher ELF magnetic field at their bottom parts are battery, CPU, and RAM memory. Furthermore, it can be assumed that the gorilla glass as a material has a lower magnetic field permeability than the plastic one. Hence, the top of the tablets is much more resistant to the magnetic field spreading than the bottom of the tablets. It is very important for a safe use of the tablets, because they are held in the users' hands. Consequently, some precautions are necessary during the tablets use. The most important suggestion is to keep a safe distance of $30 \mathrm{~cm}$ for usage. In fact, visually impaired people may tend to read text close to their eyes. This also happens with children that usually play games on tablets. Of course, this has a relation with the magnetic field decay around the tablet.

\section{CONCLUSiOnS}

The proposed study represents one of the first measurement experiments linked with the ELF magnetic field to which the tablet users are exposed. It is very important, because the tablets are very popular and spread in the younger population. Hence, the real determination of the tablets' emitted ELF magnetic field is invaluable for their users as well as for designers of the tablets. Furthermore, the measured magnetic field values were compared to the widely accepted safety standard, i.e. TCO. The results of the study showed that the level of magnetic field is typically above the safety limits. Hence, some precautions during the work with the tablets are necessary. Accordingly, an important suggestion for the safe use of the tablets was proposed.

\section{ACKNOWLEDGEMENT}

This work was partially supported by the Grant of the Ministry of Education, Science and Technological Development of the Republic of Serbia, as a part of the project TR33037.

\section{REFERENCES}

[1] Bellieni, C.V., Pinto, I., Bogi, A., Zoppetti, N., Andreuccetti, D., Buonocore, G. (2012). Exposure to elec- tromagnetic fields from laptop use of "laptop" computers. Archives of Environmental \& Occupational Health, 67(1), 31-36.

[2] ICNIRP (2010). Guidelines for limiting exposure to time-varying electric and magnetic fields (1 Hz to 100 kHz). Health Physics, 99(6), 818-836.

[3] ICNIRP (2014). Guidelines for limiting exposure to electric fields induced by movement of the human body in a static magnetic field and by time-varying magnetic fields below $1 \mathrm{~Hz}$. Health Physics, 106(3), 418-425.

[4] Gurney, J.G., Mueller, B.A., Davis, S., Schwartz, S., Stevens, R., Kopecky, K. (1996). Childhood brain tumor occurrence in relation to residential power line configurations, electric heating sources, and electrical appliance use. American Journal of Epidemiology, 143(2), 120128.

[5] Ahlbom, A., Bridges, J., De Seze, R., Hillert, L., Juutilainen, J., Mattsson, M.O., Neubauer, G., SchĂL'z, J., Simko, M. and Bromen, K. (2008). Possible effects of electromagnetic fields (EMF) on human health-opinion of the scientific committee on emerging and newly identified health risks (SCENIHR). Toxicology, 246(2-3), 248-250.

[6] COMAR. (1997). Biological and health effects of electric and magnetic fields from video display terminals. A technical information statement. IEEE Engineering in Medicine and Biology Magazine, 16(3), 87-92.

[7] TCO Development AB. (2012). TCO Certified Notebooks 4.0. http://tcodevelopment.com/files/ 2013/04/TCO-Certified-Notebooks-4. ๑. pdf

[8] Brodić, D., Amelio, A. (2015). Classification of the extremely low frequency magnetic field radiation measurement from the laptop computers. Measurement Science Review, 15(4), 202-209.

[9] Brodić, D., Amelio, A. (2016). Detecting of the extremely low frequency magnetic field ranges for laptop in normal operating condition or under stress. Measurement, 91, 318-341.

[10] Oja, M., Kaski, S., Kohonen, T. (2002). Bibliography of self-organizing map (som) papers: 1998-2001 addendum. Neural Computing Surveys, 3, 1-156.

[11] Vannucci, M., Colla, V. (2004). Meaningful discretization of continuous features for association rules mining by means of a SOM. In European Symposium on Artificial Neural Networks, 28-30 April 2004, Bruges, Belgium, 489-494. 489-494.

[12] Kohonen, T. (2001). Self-Organizing Maps. Springer Series in Information Sciences, Vol. 30.

[13] Zoppetti, N., Andreuccetti, D., Bellieni, C., Bogi, A., Pinto, I. (2011). Evaluation and characterization of fetal exposures to low frequency magnetic fields generated by laptop computers. Progress in Biophysics and Molecular Biology, 107(3), 456-463.

Received January 03, 2018. Accepted April 25, 2018. 